\title{
On the Asymptotic Behavior of $p$-Faber Polynomials for Domains with Piecewise Analytic Boundary
}

\author{
T. Tunc and M. Kucukaslan \\ Department of Mathematics, Mersin University, 3343 Mersin, Turkey \\ Correspondence should be addressed to M. Kucukaslan, mkkaslan@gmail.com \\ Received 3 July 2011; Accepted 8 August 2011 \\ Academic Editor: X.-L. Gao
}

Copyright (c) 2011 T. Tunc and M. Kucukaslan. This is an open access article distributed under the Creative Commons Attribution License, which permits unrestricted use, distribution, and reproduction in any medium, provided the original work is properly cited.

Let $G \subset \mathbb{C}$ be a domain bounded by a piecewise analytic Jordan's curve $L$, and let $F_{n, p}$ denote the $p$-Faber polynomials associated with $G$. We derive estimates of the form $F_{n, p}(z)=O\left(1 / n^{\eta}\right)$, $(n \rightarrow \infty)$ for $z \in G$, where $\eta$ depends on geometric properties of $L$ and the parameter $p$. Also, we show that $O$ cannot be replaced by $o$ in the relation given above.

\section{Introduction and Main Results}

Let $G \subset \mathbb{C}$ be a domain bounded by a Jordan curve $L$, and let $\Phi$ be the conformal mapping of the domain $\Omega:=\mathbb{C}_{\infty} \backslash \bar{G}$, where $\mathbb{C}_{\infty}:=\mathbb{C} \cup\{\infty\}$, onto $\Delta:=\{w:|w|>1\}$ with the usual normalization at infinity:

$$
w=\Phi(z)=\alpha z+a_{0}+\frac{a_{1}}{z}+\cdots, \quad \alpha>0, z \in \Omega .
$$

Let $\Psi:=\Phi^{-1}: \Delta \rightarrow \Omega$ denote the inverse conformal map. Then,

$$
z=\Psi(w)=\beta w+b_{0}+\frac{b_{1}}{w}+\cdots, \quad|w|>1,
$$

where $\beta=1 / \alpha$ gives the capacity $\operatorname{cap}(L)$ of $L$. 
Let $0<p<\infty$. The $p$-Faber polynomials $F_{n, p}$ associated the domain $G$ are defined as the polynomial part of the expansion of

$$
\Phi^{n}(z)\left(\Phi^{\prime}(z)\right)^{1 / p}, \quad n=0,1,2, \ldots
$$

in a neighborhood of the infinity. Therefore, from (1.1) we have

$$
\Phi^{n}(z)\left(\Phi^{\prime}(z)\right)^{1 / p}:=F_{n, p}(z)+E_{n, p}(z), \quad z \in \Omega
$$

where

$$
F_{n, p}(z):=\alpha^{n+1 / p} \quad z^{n}+\cdots
$$

is the $p$-Faber polynomial of degree $n$ and

$$
E_{n, p}(z)=\frac{c_{0}}{z}+\frac{c_{1}}{z^{2}}+\frac{c_{2}}{z^{3}}+\cdots
$$

is the singular part of $\Phi^{n}(z)\left(\Phi^{\prime}(z)\right)^{1 / p}$. Indeed, the number $c_{0}$ vanishes, since $z\left(\left(\Phi^{\prime}(z)\right)^{1 / p}-\right.$ $\left.\alpha^{1 / p}\right)$ tends to zero when $z$ tends to infinity. Also, the $p$-Faber polynomials associated with $G$ can be defined by the generating function

$$
g(w):=\frac{\left(\Psi^{\prime}(w)\right)^{1-1 / p}}{\Psi(w)-z}=\sum_{n=0}^{\infty} \frac{F_{n, p}(z)}{w^{n+1}}, \quad z \in G,|w|>1
$$

see $[1,2]$.

If $p$ tends to infinity, the $p$-Faber polynomials coincide with the usual Faber polynomials $F_{n}$. If $p=1$, then it follows immediately from (1.4) that

$$
F_{n, 1}(z)=\frac{F_{n+1}^{\prime}(z)}{n+1}
$$

where $F_{n}$ is the Faber polynomials of $\mathrm{G}$ of degree $n$.

The aim of this paper is to find asymptotic behaviors of $p$-Faber polynomials $F_{n, p}$ in $G$ and $E_{n, p}$ in $\Omega$.

If $L$ is rectifiable, then $\Phi^{\prime}$ belongs to the Smirnov class $E^{1}(\Omega\{\infty\})$. In addition, both $\Psi^{\prime}$ and $\Phi^{\prime}$ have nontangential limits almost everywhere on $\mathbb{T}$ boundary of the unit disc $\mathbb{D}:=$ $\{w \in \mathbb{C}:|w|<1\}$ and $L$; respectively, and they are integrable with respect to the arc length measure; see [3] or [4]. 
Assume now that the boundary $L$ is rectifiable. For such a boundary, Cauchy's integral formula yields the following representations for the $p$-Faber polynomials and their associated singular parts:

$$
\begin{array}{ll}
F_{n, p}(z)=\frac{1}{2 \pi i} \int_{L} \frac{\Phi^{n}(\zeta)\left(\Phi^{\prime}(\zeta)\right)^{1 / p}}{\zeta-z} d \zeta, & z \in G, \\
E_{n, p}(z)=\frac{1}{2 \pi i} \int_{L} \frac{\Phi^{n}(\zeta)\left(\Phi^{\prime}(\zeta)\right)^{1 / p}}{\zeta-z} d \zeta, & z \in G .
\end{array}
$$

Our assumption for this work is that $L$ is a piecewise analytic Jordan's curve. That is, $L=$ $\bigcup_{j=1}^{m} L_{j}, m \in \mathbb{N}$, where $L_{j}$ are a finite number of analytic Jordan's arcs meeting at corners $z_{j}$ where $L$ has an exterior angle $\lambda_{j} \pi$ with $0<\lambda_{j}<2$. Cusps are, therefore, excluded, but $\lambda_{j}=1$ may occur-a "smooth corner".

Let $\lambda:=\min _{j=1, \ldots, m}\left\{\lambda_{j}\right\}$. Gaier [5] proved that

$$
F_{n}(z)=O\left(\frac{1}{n^{\lambda}}\right) \quad(n \rightarrow \infty)
$$

for $z \in G$, uniformly on compact subsets of G. Stylianopoulos [6] indicated that one can obtain

$$
F_{n, 1}(z) \leq \frac{c(L, F)}{n^{\lambda+1}}, \quad z \in F
$$

by using (1.8) and (1.13), where $F$ is an arbitrary compact subset of $G$, and $c(L, F)$ is a constant depending on $L$ and $F$. Estimates of the form (1.13) have been given by Suetin [2, 7], for boundary $L$ has high degree of smoothness.

For simplicity of notation, we write

$$
\eta:=\eta(\lambda, p):=(1-\lambda)\left(1-\frac{1}{p}\right)
$$

It is clear that, if $0<\lambda<2$ and $1 / 2 \leq p \leq \infty$, then $|\eta|<1$.

The main results of the paper are the following.

Theorem 1.1. Let $1 / 2 \leq p \leq \infty$, and let $G \subset \mathbb{C}$ be bounded by a piecewise analytic Jordan curve $L$. If $L$ has exterior angles $\lambda_{j} \pi$ with $0<\lambda_{j}<2$ and if $\lambda:=\min _{j} \lambda_{j}$, then

$$
F_{n, p}(z)=O\left(\frac{1}{n^{1-\eta}}\right) \quad(n \rightarrow \infty)
$$

for $z \in G$, uniformly on compact subsets of $G$.

Remark 1.2. From the proof of Theorem 1.1, it follows that an estimate of the form (1.13) holds also all derivatives of the $p$-Faber polynomials. 
Remark 1.3. If $L$ has no smooth corners; that is, all $\lambda_{j} \neq 1, O$ cannot be replaced by $o$ in (1.13).

Remark 1.4. If $p$ tends to infinity, we get the result (1.13) of Gaier.

Similar arguments in the proof of Theorem 1.1 provide the following theorem.

Theorem 1.5. Under the assumptions of Theorem 1.1, we have, for any $n \in \mathbb{N}$,

$$
\left|E_{n, p}(z)\right| \leq \frac{c(L)}{\operatorname{dist}(z, L)} \frac{1}{n^{1-\eta}}, \quad z \in \Omega,
$$

where $c(L)$ depends on L only.

Remark 1.6. The equalities (1.13) and (1.14) are also valid for $0<p<1 / 2$ under condition $\max _{j=1, \ldots, m} \lambda_{j}<1 /(1-p)$.

Remark 1.7. If $p=1$, we get the result of Stylianopoulos [6]:

$$
\left|E_{n, 1}(z)\right| \leq \frac{c(L)}{\operatorname{dist}(z, L)} \frac{1}{n}, \quad z \in \Omega
$$

We will prove Theorems 1.1, 1.5 and Remark 1.3 in section 3.

\section{Auxiliary Results}

We are going to follow the analog used by Gaier in [5]. Since the components $L_{j}$ of $L$ are assumed to be analytic Jordan's arcs, the mapping $\Phi$ from $\Omega$ to $\Delta$ can be continued analytically beyond each point of $L$ that is not corner; this follows from Schwarz's reflection principle. Since $L$ has no cusps, we can refine this statement. For each $j=1,2, \ldots, m$, there are (short) line segments $s_{j}$ and $s_{j}^{\prime}$ in the interior of $L$ meeting $L_{j}$ at $z_{j}$ and $z_{j+1}$, respectively, at a (small) positive angle and a rectifiable arc $\gamma_{j}$ lying entirely in the interior of $L$ and connecting the other two endpoints of $s_{j}$ and $s_{j}^{\prime}$ such that $\Phi$ has analytic continuation beyond $L_{j}$ into the strip-like domain $g_{j}$ bounded by $L_{j}, s_{j}, s_{j}^{\prime}$, and $\gamma_{j}$ (see [5] or [6] for figure). Therefore, the $\operatorname{arc} \Phi\left(\gamma_{j}\right)$ is in $\mathbb{D}$ while $\Phi\left(s_{j}\right)$ and $\Phi\left(s_{j}^{\prime}\right)$ are arcs in $\mathbb{D}$ except for their endpoints $\Phi\left(z_{j}\right)$ and $\Phi\left(z_{j+1}\right)$. More can be said: since reflection preserves angles and since the exterior angle at $z_{j}$ of opening $\lambda_{j} \pi$ is mapped onto a angle of opening $\pi$ at $\Phi\left(z_{j}\right)$, we see that $\Phi\left(s_{j}\right)$ lies in a Stolz angle in $\mathbb{D}$ with corner at $\Phi\left(z_{j}\right)$ :

$$
\frac{\left|\Phi(z)-\Phi\left(z_{j}\right)\right|}{1-|\Phi(z)|} \leq c_{1} \quad \text { for } z \in s_{j}, \quad j=1,2, \ldots, m
$$

and similarly

$$
\frac{\left|\Phi(z)-\Phi\left(z_{j+1}\right)\right|}{1-|\Phi(z)|} \leq c_{1}^{\prime} \quad \text { for } z \in s_{j}^{\prime}, \quad j=1,2, \ldots, m
$$


Furthermore, we have the following inequalities for $z$ on $s_{j}(j=1,2, \ldots, m)$ :

$$
\begin{gathered}
\left|\Phi(z)-\Phi\left(z_{j}\right)\right| \geq \text { const } \cdot\left|z-z_{j}\right|^{1 / \lambda_{j}}, \\
\left|\Phi^{\prime}(z)\right| \leq \text { const } \cdot\left|z-z_{j}\right|^{1 / \lambda_{j}-1}, \\
|\Phi(z)| \leq 1-\text { const } \cdot\left|z-z_{j}\right|^{1 / \lambda_{j}}, \\
\operatorname{dist}(z, L) \geq \text { const } \cdot\left|z-z_{j}\right| .
\end{gathered}
$$

The similar inequalities of (2.3)-(2.6) can be written by replacing $s_{j}, z_{j}$, and $\lambda_{j}$ with $s_{j}^{\prime}, z_{j+1}$, and $\lambda_{j+1}$, respectively. The inequalities (2.3) and (2.4) emerge from Lehman's asymptotic expansions of conformal mappings near an analytic corner [8]. The inequality (2.5) follows from (2.3), because reflection preserves angles. Finally, (2.6) is a simple fact of conformal mapping geometry.

The last auxiliary facts are on the behavior of $g$ near $\mathbb{T}$ obtained by using Lehman's asymptotic expansions of conformal mappings near an analytic corner [8]. Assume that $L$ has a corner at $\zeta$ with exterior angle $\lambda \pi, 0<\lambda<2$ and that $\zeta=\Psi(1)$. Let further $z \in G$ be fixed. Similar arguments in [5] apply to the $g(w)=\left(\Psi^{\prime}(w)\right)^{1-1 / p} /(\Psi(w)-z)$ one can obtain that if $0<\lambda<1$, then

$$
g(w) \cong \frac{C}{(w-1)^{\eta}}, \quad \text { as } w \rightarrow 1+, \text { with } C \neq 0 .
$$

If $1<\lambda<2$, then

$$
g^{\prime}(w) \cong \frac{C}{(w-1)^{\eta+1}}, \quad \text { as } w \rightarrow 1+, \text { with } C \neq 0 .
$$

\section{Proofs of Theorems}

From now on, $c$ denote positive constants, not necessarily the same at the different places and not depending on $n$ degree of the $p$-Faber polynomial and the parameter $p$. We recite that

$$
\eta:=(1-\lambda)\left(1-\frac{1}{p}\right)
$$

Proof of Theorem 1.1. Here we use the integral representation (1.9) of the $p$-Faber polynomials $F_{n, p}$ in $G$. Given a compact subset $F$ of $G$, we deform the path of integration by replacing each 
component $L_{j}$ of $L$ by some $L_{j}^{\prime}:=s_{j} \cup L_{j} \cup s_{j}(j=1,2, \ldots, m)$, as mentioned before, with proper orientation and such that $F \subset L^{\prime}:=\bigcup_{j=1}^{m} L_{j}^{\prime}$. This gives

$$
\begin{aligned}
\left|F_{n, p}(z)\right| & \leq \frac{1}{2 \pi} \sum_{j=1}^{m} \int_{L_{j}^{\prime}} \frac{|\Phi(\zeta)|^{n}\left|\Phi^{\prime}(\zeta)\right|^{1 / p}}{|\zeta-z|}|d \zeta| \\
& \leq C(L, F) \sum_{j=1}^{m} \int_{L_{j}^{\prime}}|\Phi(\zeta)|^{n}\left|\Phi^{\prime}(\zeta)\right|^{1 / p}|d \zeta| \\
& =C(L, F) \sum_{j=1}^{m}\left(\int_{s_{j}}+\int_{s_{j}^{\prime}}+\int_{L_{j}}\right)|\Phi(\zeta)|^{n}\left|\Phi^{\prime}(\zeta)\right|^{1 / p}|d \zeta| .
\end{aligned}
$$

The contributions of the arcs $L_{j}$ to these integrals are obviously $\mathrm{O}\left(\rho^{n}\right)$ for some $\rho<1$, so that typical integrals

$$
I_{j}=\int_{s_{j}}|\Phi(\zeta)|^{n}\left|\Phi^{\prime}(\zeta)\right|^{1 / p}|d \zeta|
$$

extended over the line segments $s_{j}$ (and $s_{j}^{\prime}$ ) remain to be estimated. If $s$ is arc length of $s_{j}$ measured from $z_{j}$, by using (2.4) and (2.5),

$$
|\Phi(\zeta)| \leq 1-c s^{1 / \lambda_{j}}<\exp \left(-c s^{1 / \lambda_{j}}\right), \quad\left|\Phi^{\prime}(\zeta)\right| \leq c s^{1 / \lambda_{j}-1},
$$

and, therefore,

$$
\begin{aligned}
I_{j} & <c \int_{0}^{\infty} \exp \left(-c n s^{1 / \lambda j}\right) s^{\left(1 / \lambda_{j}-1\right) / p} d s \\
& =\frac{c}{n^{\left(1-\lambda_{j}\right) / p+\lambda_{j}}} \int_{0}^{\infty} e^{-t} t^{(1 / p-1)\left(1-\lambda_{j}\right)} d s .
\end{aligned}
$$

If $1 / 2 \leq p \leq \infty$, then the integral in the last line is convergent; that is,

$$
I_{j}<\frac{c}{n^{\left(1-\lambda_{j}\right) / p+\lambda_{j}}}
$$

From this we obtain the Theorem 1.1.

In the case $0<p<1 / 2$, similar arguments show that, under the condition $\lambda_{j}<1 /(1-$ $p)$, the inequality (3.6) is valid. This proves Remark 1.4.

Proof of Remark 1.3. We now show that $O$ in Theorem 1.1 cannot be replaced by $o$. Let $L$ be any piecewise analytic Jordan's curve that has no smooth corners; that is, all $\lambda_{j} \neq 1$. We assume that one of them is at $\Psi(1)$ with exterior angle $\lambda$. Assume that $F_{n, p}(z)=o\left(1 / n^{1-\eta}\right)(n \rightarrow \infty)$ for some fixed $z \in G$. 
(a) If $0<\lambda<1$ and $p>1$, we consider for $|w|>1$

$$
g(w)=\frac{\left(\Psi^{\prime}(w)\right)^{1-1 / p}}{\Psi(w)-z}=\sum_{n=0}^{\infty} \frac{F_{n, p}(z)}{w^{n+1}} .
$$

Since

$$
\sum_{n=1}^{\infty} \frac{x^{n}}{n^{\alpha}} \sim \frac{\Gamma(1-\alpha)}{(1-x)^{1-\alpha}}, \quad \text { when } x \rightarrow 1-
$$

for $\alpha<1$, where $\Gamma$ is Gamma Function (see [9, page 225]), then

$$
|g(w)|=o\left(\sum_{n=1}^{\infty} \frac{1}{n^{1-\eta} w^{n}}\right)=o(1) \frac{1}{(w-1)^{\eta}}, \quad \text { as } w \rightarrow 1+.
$$

This contradicts (2.7).

(b) If $1<\lambda<2$, then, by using (3.8) and

$$
g^{\prime}(w)=-\sum_{n=0}^{\infty} \frac{(n+1) F_{n, p}(z)}{w^{n+2}}
$$

we get

$$
\left|g^{\prime}(w)\right|=o\left(\sum_{n=1}^{\infty} \frac{n}{n^{1-\eta} w^{n}}\right)=o(1) \frac{1}{(w-1)^{\eta+1}}, \quad \text { as } w \rightarrow 1+
$$

which is contradiction to (2.8).

Proof of Theorem 1.5. The proof goes along similar lines as those taken in the proof of Theorem 1.1 with one significant difference: here, $z$ lies in $\Omega$, instead of $G$, and, thus, can tend to $L$ without need altering the curve $L^{\prime}$. As a consequence, the set $F$ defined above does not depend on $z$, and; thus, $\operatorname{dist}(z, F)>\operatorname{dist}(L, F)=c(L)$.

\section{References}

[1] D. M. Israfilov, "Approximation by p-Faber polynomials in the weighted Smirnov class $E^{P}=(G, \omega)$ and the Bieberbach polynomials," Constructive Approximation, vol. 17, no. 3, pp. 335-351, 2001.

[2] P. K. Suetin, Series of Faber Polynomials, Gordon and Breach Science Publishers, Amsterdam, The Netherlands, 1998.

[3] P. L. Duren, Theory of $H^{p}$ Spaces, vol. 38 of Pure and Applied Mathematics, Academic Press, New York, NY, USA, 1970.

[4] C. Pommerenke, Boundary Behaviour of Conformal Maps, Springer, Berlin, Germany, 1992.

[5] D. Gaier, "On the decrease of Faber polynomials in domains with piecewise analytic boundary," Analysis, vol. 21, no. 2, pp. 219-229, 2001.

[6] N. Stylianopoulos, "Strong asymptotics for Bergman polynomials over domains with corners," http://arxiv.org/abs/0910.1788v2. 
[7] P. K. Suetin, "The basic porperties of Faber polynomials," Uspekhi Matematicheskikh Nauk, vol. 19, no. 4, pp. 125-154, 1964, English translation in Russian Mathematical Surveys, vol. 19, no. 4, pp. 121-149, 1964.

[8] R. S. Lehman, "Development of the mapping function at an analytic corner," Pacific Journal of Mathematics, vol. 7, pp. 1437-1449, 1957.

[9] E. C. Titchmarsh, The Theory of Functions, Oxford University Press, Oxford, UK, 2nd edition, 1993. 


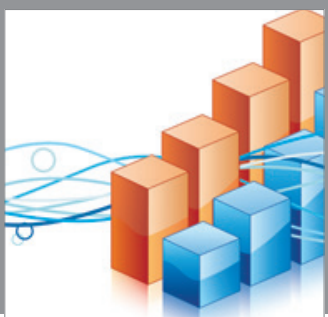

Advances in

Operations Research

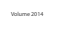

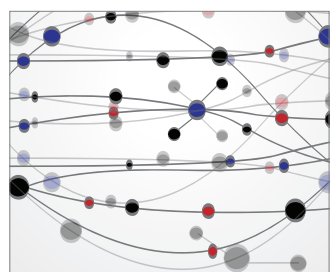

\section{The Scientific} World Journal
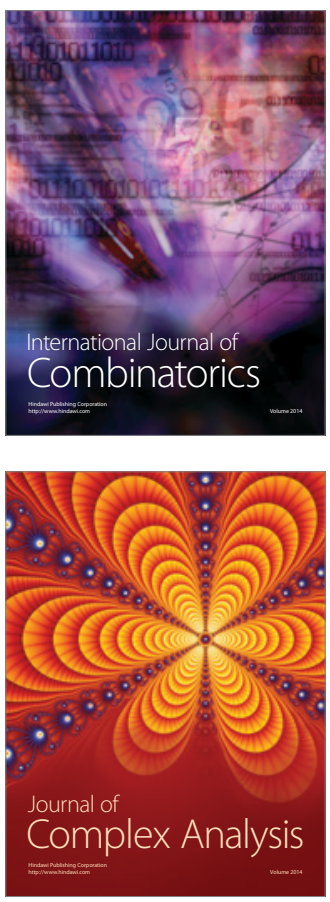

International Journal of

Mathematics and

Mathematical

Sciences
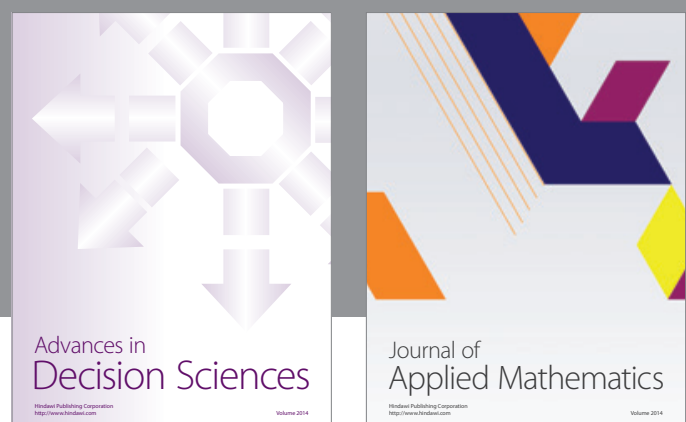

Journal of

Applied Mathematics
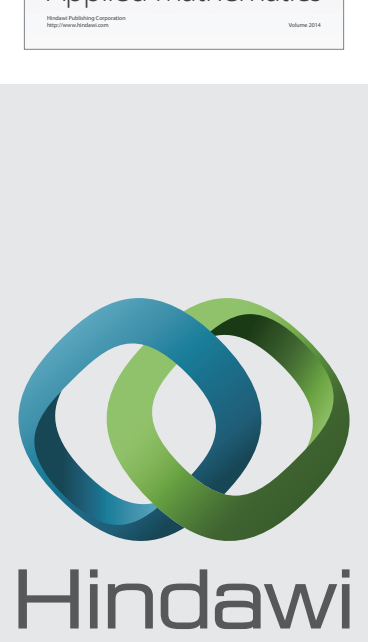

Submit your manuscripts at http://www.hindawi.com
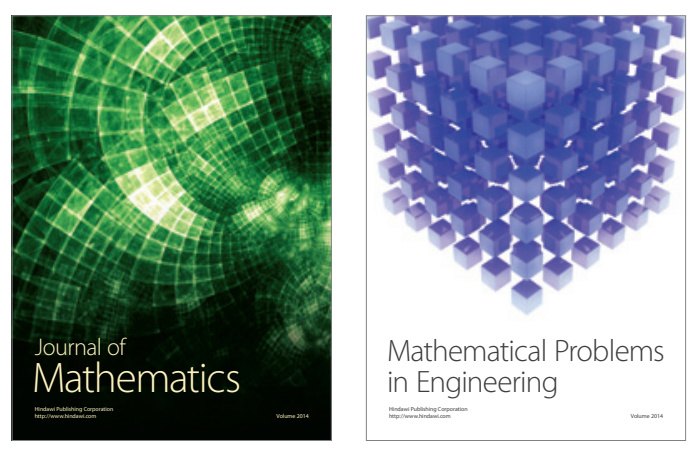

Mathematical Problems in Engineering
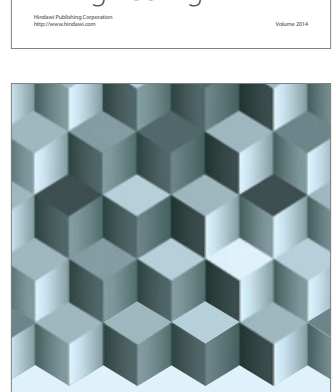

Journal of

Function Spaces
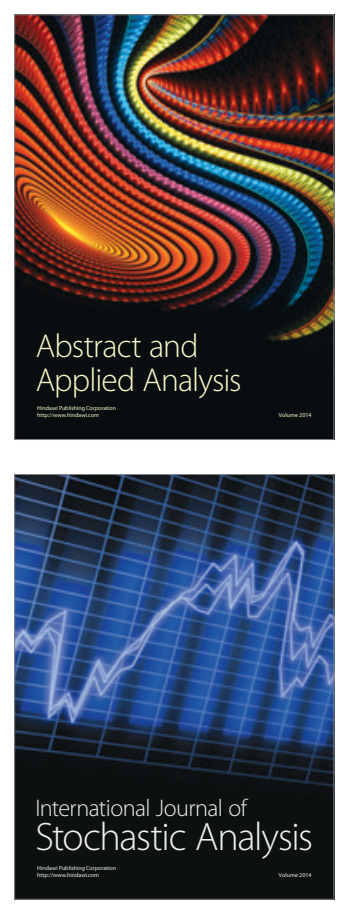

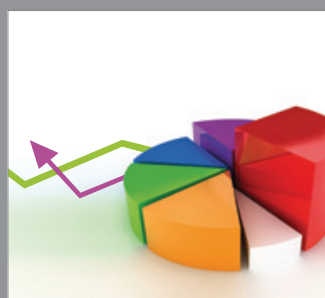

ournal of

Probability and Statistics

Promensencen
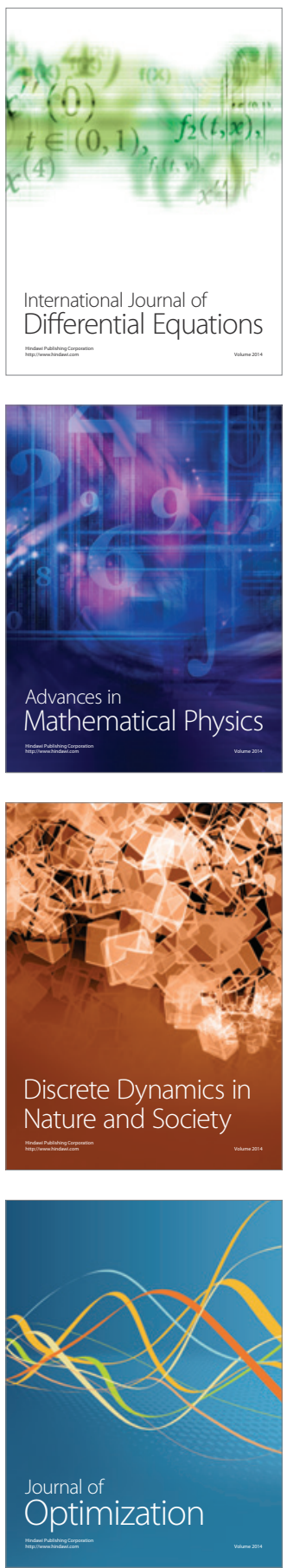